\title{
ON SOME RINGS WHOSE MODULES HAVE MAXIMAL SUBMODULES
}

\author{
V. P. CAMILLO
}

ABSTRACT. It is shown that a principal right ideal domain, having the property that every right $R$ module has a maximal submodule must be simple. Strong conditions satisfied by these rings are deduced giving evidence for the conjecture that they must be $V$-rings. We also generalize an example of Faith by showing that a subring of an infinite dimensional full linear ring, which contains the socle of that ring is never a left $V$-ring.

Cozzens [1] and Koifmann [3] gave striking examples of simple principal right ideal domains which are right $V$-rings (every simple module is injective). These examples answer two questions of Faith, whether there exists a nonsemisimple, simple, noetherian $V$-ring, and whether every $V$-ring is regular; and Bass' question, whether a ring $R$ over which every right $R$ module has a maximal submodule ( $\max$ ring) must be right perfect. Interest in these examples is enhanced by the fact that, being simple domains, they are highly nonregular and highly nonperfect, thus answering the last two questions, very reasonable ones given the state of knowledge at the time, very definitively in the negative. They show that in the noncommutative case (see [3] for the commutative case) the hypothesis " $R$ is a max ring" does not seem to place very strong conditions on $R$.

Now the most general proof of the fact that these rings are simple comes from a theorem of Faith [2, p. 130], that any $V$-ring which is an order in a semisimple ring must be a product of simple rings. Our first purpose is to show that in fact if $R$ is a right P.I.D., as the examples cited are, then one needs only assume that $R$ is a right max ring in order to conclude that $R$ is simple. This result seems interesting because it seems to be an exception to the apparent weakness of the max ring hypothesis as cited in the previous paragraph.

Received by the editors April 19, 1974 .

AMS (MOS) subject classifications (1970). Primary 16A04, 16A30; Secondary 16A42, 16A46. $V$-ring.

Key words and phrases. Maximal submodule, principal ideal ring, simple ring, 
The question that naturally arises from this is: Are these rings simple because they are $V$-rings? This problem seems to be quite difficult. We are able, however, to present some strong evidence for its truth. We show that if $R$ is a right P.I.D. and a right max ring then for all $a, b \in R$ which generate maximal right ideals, either $R / a b R$ or $R / b a R$ is semisimple. This is interesting because, as is explained in the text (before Proposition 2), if $R$ is also a left P.I.D. then $R$ is a $V$-ring if and only if $R / a b R$ is semisimple for all such $a$ and $b$.

The second section is motivated by the example given by Faith of a regular ring which is not a $V$-ring. (A commutative ring is a $V$-ring if and only if it is regular.) Faith's example is the subring of any full linear ring $L=$ End $V_{F}, F$ a field, generated by the socle of $L$ and the scalars. We generalize this by showing directly that if $L=$ End $V_{D}, D$ a division ring, and if $R$ is any subring of $L$ containing socle $L$, then $R$ is not a left $V$-ring.

I. Theorem 1. If $R$ is a right max ring, which is a right P.I.D., then $R$ is simple.

Proof. Let $d R$ be a two-sided ideal in $R$, and let $Q$ be the classical right quotient ring of $R$. Let $U \subset Q$ be given by $U=\bigcup_{n \in N} d^{-n} R$. Then $U / R$ has a maximal submodule $M / R$. Now, there is an $n$ with $d^{-n+1} \in M$, but $d^{-n} \notin M$. However, since $U / M$ is simple, $d^{-n-1}+M=d^{-n} r+M$ for some $r \in R$. But $r d=d s$ for some $s$, so that $d^{-1} r=s d^{-1}$. Thus, $d^{-n-1}+$ $M=d^{-n+1} s d^{-1}+M$ and multiplying on the right by $d$ we have $d^{-n}+M=$ $d^{-n+1} s+M$.

However, the right side is zero since $d^{-n+1} \in M$, so $d^{-n} \in M$. This shows that $U / R=0$, or that $d^{-1} \in R$, so that $d R=R$.

The previous result suggests the following: If $R$ is a P.I.D., is $R$ a $V$-ring if and only if it is a max ring? This question seems difficult. Below, however, we present a proposition which is quite suggestive. The reason for this is that if $R$ is a two-sided P.I.D. one can show in a relatively straightforward manner that $R$ is a right $V$-ring if and only if $R / a b R$ is semisimple for all $a$ and $b$ which generate maximal right ideals. (Just determine that the injective hull of a simple module must have a submodule of length two, which must be of the form $R / a b R$.)

Proposition 2. Let $R$ be a right max ring which is also a right Ore domain with classical right quotient ring $Q$. Then:

(a) If $a, b \in R$ and $a$ and $b$ generate maximal right ideals, either $R / a b R$ or $R / b a R$ is semisimple. 
(b) If $a R$ is a maximal right ideal then $R / a^{n} R$ is semisimple for all $n \in N$.

Proof. (a) Use the same idea as in the proof of Theorem 1. Consider the chain $a^{-1} R \subset a^{-1} b^{-1} R \subset a^{-1} b^{-1} a^{-1} R \ldots$ in $Q$, and let $U$ be the union. Let $M / R$ be a maximal submodule of $U / R$. Then there is an $n$ such that either:

(1) $\left(a^{-1} b^{-1}\right)^{n-1} \in M$ but $\left(a^{-1} b^{-1}\right)^{n-1} a^{-1} \notin M$, or

(2) $a^{-1}\left(b^{-1} a^{-1}\right)^{n-1} \in M$ but $a^{-1}\left(b^{-1} a^{-1}\right)^{n-1} b^{-1}=\left(a^{-1} b^{-1}\right)^{n} \notin M$.

If (1) occurs, let $x=\left(a^{-1} b^{-1}\right)^{n}$. Then $(x: M)=\{r \in R \mid x r \in M\}$ is a maximal right ideal in $R$ which contains $b a$. It does not contain $b$, or else $\left(a^{-1} b^{-1}\right)^{n-1} a^{-1} \in M$. Thus

$$
R / b a R=b R / b a R \oplus(x: M) / b a R .
$$

If case (2) occurs, an analogous argument shows that $R / a b R$ is semisimple.

(b) Again let $U=\bigcup_{a \in N} a^{-n} R$ and let $M / R$ be a maximal submodule of $U / R$. Then there is a $k$ such that $a^{-k} \notin M$, but $a^{-k+1} \in M$. Look at $C=\left(a^{-m-k}: M\right)$. Then $C$ is a maximal right ideal containing $a^{n+1}$ but not $a^{n}$. Since $a^{n} R / a^{n+1} R \quad R / a R$ is simple we have

$$
R / a^{n+1} R=a^{n} R / a^{n+1} R \oplus C / a^{n+1} R .
$$

Then, $C / a^{n+1} R \approx R / a^{n} R$, which we may assume to be semisimple by induction, so that $R / a^{n+1} R$ is.

Remarks. (A) Proposition 2 may be proved under the hypothesis that $R$ is a semiprime principal right ideal ring.

(B) Theorem 1 also works for a prime Goldie ring which is right principal since we only require that the ideal in question be generated by a regular element.

(C) If $R$ is a principal right ideal domain with a unique simple module then, in fact, $R$ is a right max ring if and only if $R$ is a right $V$-ring, for the right quotient ring $Q$ of $R$ is injective and has a maximal submodule $M$. Then $Q / M$ is injective since $R$ is hereditary. The idea for this is found in [3, Proposition 1.1].

II. Writing functions opposite scalars we have the following generalization of Faith's example, as explained in the introduction. Recall the socle of a full linear ring is a collection of all linear transformations of finite rank, a fact we use without explicit mention below. 
Theorem 3. If $R$ is a subring of an infinite dimensional full linear ring $L=$ End $W_{D}$, containing socle $L$, then $R$ is not a left $V$-ring.

Proof. Let $X$ be a basis and pick out countable subset $\left\{x_{i} \mid x_{i} \in N\right\}$. For each $i \in N$ let $f_{i} \in R$ be defined by $f_{i}\left(x_{i}\right)=x_{1}$ and $f_{i}(x)=0$ for $x \in X$; $x \neq x_{i}$. Then $\Sigma R f_{i}$ is direct, for if $p_{1} f_{1}+\cdots+p_{n} f_{n}=0$ then, for any $k$ we have

$$
p_{1} f_{1}\left(x_{k}\right)+\cdots+p_{k} f_{k}\left(x_{k}\right)+\cdots+p_{n} f_{n}\left(x_{k}\right)=0
$$

and, since $p_{i} f_{i}\left(x_{k}\right)=0$ for $i \neq k$, we have $p_{k} f_{k}\left(x_{k}\right)=0$ and thus, $p_{k} f_{k}(x)=$ 0 for all $x \in X$ so that $p_{k} f_{k}=0$.

Further, if $l(g)$ denotes the left annihilator of $g \in L$, then $l\left(f_{k}\right) \subset l\left(f_{1}\right)$. In fact, if $\operatorname{Im} L_{1} \supset \operatorname{Im} L_{2}$ for any two linear transformations then $l\left(L_{1}\right) \subset$ $l\left(L_{2}\right)$, for if $p L_{1}=0$, then for any $x \in W, L_{2}(x)=L_{1}(y)$ so that $p L_{2}(x)=$ $p L_{1}(y)=0$ and $p L_{2}=0$.

Thus, the maps $H_{i}$ defined by $\left(p f_{i}\right) H_{i}=p f_{1}$ are well defined. Since $\Sigma R f_{i}$ is direct, this yields a map $H: \Sigma R f_{i} \rightarrow R f_{1}$ given by

$$
\left(\sum p_{i} f_{i}\right) H=\sum\left(p_{i} f_{i}\right) H_{i}=\left(\sum p_{i}\right) f_{1} \text {. }
$$

Now, $R f_{1}$ is simple. For if $p f_{1} \neq 0$ then $p f_{1}\left(x_{1}\right) \neq 0$ so there is a linear transformation $g$ with $g p f_{1}\left(x_{1}\right)=x_{1}$ and $\operatorname{Im} g \subset x_{1} D$. Then $g \in R$ since $R$ contains socle $L$. But clearly, from the construction of $f_{1}, g p f_{1}=$ $f_{1}$ so that $R p f_{1}=R f_{1}$ and thus $R f_{1}$ is simple.

We now show that the map $H$ cannot be extended and that $R f_{1}$ is therefor a simple noninjective module. For, let (1) $H=t f_{1}$ and let $t f_{1}\left(x_{1}\right)=$ $x_{1} d_{1}+\cdots+x_{n} d_{n}$. Then, $f_{1}=\left(f_{n+1}\right) H=f_{n+1} t f_{1}$ so that $f_{1}=f_{n+1} t f_{1}$ or $f_{1}\left(x_{1}\right)=f_{n+1} t f_{1}\left(x_{1}\right)$ or

a contradiction.

$$
x_{1}=f_{n+1}\left(\sum_{i=1}^{n} x_{i} d_{i}\right)=\sum_{i=1}^{n} f_{n+1}\left(x_{i}\right) d_{i}=0
$$

\section{REFERENCES}

1. J. Cozzens, Homological properties of the ring of differential polynomials, Bull. Amer. Math. Soc. 76 (1970), 75-79. MR 41 \#3531.

2. C. C. F aith, Lectures on injective modules and quotient rings, Lecture Notes in Math., no. 49, Springer-Verlag, Berlin and New York, 1967. MR 37 \#2791.

3. L. A. Koifmann, Rings over which each module has a maximal submodule, Mat. Zametki 7 (1970), 359-367 = Math. Notes 7 (1970), 215-219. MR 41 \#6913. 